\title{
Wer die Wahl hat, hat die Qual
}

Erhard Taverna

erhard.taverna[at]saez.ch
Fortschritte haben auch Nebenwirkungen. Zum Beispiel, wenn eine Patientin über das weitere Vorgehen entscheiden soll: brusterhaltend oder doch Ablatio, sofortiger oder späterer Aufbau, Wahl der Chemotherapie. Jede Option erfordert eine neue Entscheidung, ein Prozess zwischen Hoffnung und Überforderung. Verständliche und klare Informationen sind gefragt und oft auch Unterstützung während der vorgegebenen Bedenkzeit. Eine gemeinsame Entscheidungsfindung (shared decision making) braucht Vertrauen, Zeit, Erfahrung und Wissen.

Liselotte Dietrich, geboren 1962, ist eine erfahrene Spezialistin für die schwierigen Begleitumstände, die sich aus der zunehmenden Behandlungs-Komplexität ergeben. Nach der Matura arbeitete sie mehrere Jahre als diplomierte Pflegefachfrau am Inselspital Bern. Sie setzte ihre Arbeit bei der Krebsliga Bern fort, nahm an einem Projekt des Nationalfonds teil und liess sich berufsbegleitend als Sozialbegleiterin ausbilden. Dem Aufbau der ambulanten psychoonkologischen Beratung am Kantonsspital St. Gallen folgte die Ausbildung zur Supervisorin, seit 2006 arbeitet sie in eigener Praxis in Herisau. Die Hälfte der Arbeitszeit verbringt die Fachfrau als Ausbildnerin an Tagungen, Seminaren und Schulungen in Spitälern, Behindertenheimen und Praxen. Pflegende und Ärzte werden an Fallsituationen geschult, etwa im Übermitteln schlechter Nachrichten oder in der Begleitung Sterbender und deren Angehörigen. Seit 2002 ist zur Erlangung des FMH-Titels Onkologie ein Kommunikationstraining vorgeschrieben.

Frau Dietrich ist Mitglied einer Expertengruppe, die von der Krebsliga Schweiz für diese Kurse aufgeboten wird. Oft seien es unverfängliche, administrative Massnahmen, die unvorhergesehene Konsequenzen nach sich zögen. Wenn ein Spital bei jedem Patienteneintritt nach einer Patientenverfügung frage, gehe es letztlich immer auch um das Sterben. Dann genüge es nicht, sich nur auf Sachinhalte zu konzentrieren, denn wichtiger sei die emotionale Ebene, der individuelle Denkprozess: «Was heisst für Sie leiden?» Auch die informierte Zustimmung (informed consent) sei eine gute Gelegenheit für Gespräche, die über die juristische Absicherung hinausgehen. Dazu gibt es zahlreiche weitere Beispiele, weil der Mehraufwand bei zusätzlichen Angeboten häufig unterschätzt werde. Etwa Fragen im Rahmen der Palliation, der Einbezug fremder Kulturen, ein Anamneseformular zum Glauben. Ist es sinnvoll, enge Verwandte als Dolmetscher einzusetzen, wie erleben Folteropfer ein Spital,

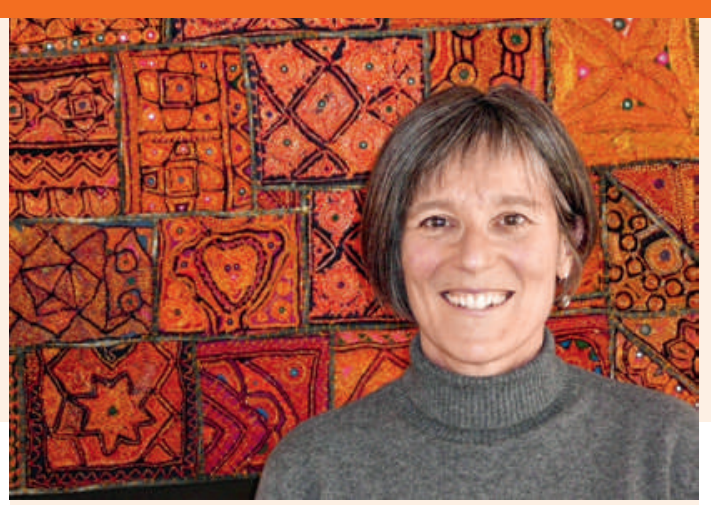

Liselotte Dietrich: Spezialistin für gelingende Kommunikation zwischen Ärzten und Patienten und für Unterstützung bei schwierigen Entscheidungen.

wie wirkt sich der konfrontativ-autoritäre Auftritt mancher Ärzte aus, wie geht man mit ungewohnten, patriarchalen Rollenbildern um? Es genügt nicht, einen Fragenkatalog einfach abzuhaken. Oft strapaziert die theoretisch geforderte, totale, gegenseitige Transparenz die Kranken. «Ich will gar nicht im Voraus alles wissen», sei eine häufig vorgebrachte Haltung, die zu akzeptieren sei. Denn die Lebensqualität wird beeinflusst durch das Gleichgewicht von Erwartung und Realität. In diesem Kontext könne auch eine bewusste Verdrängung als sinnvolle, realistische Anpassung die Zufriedenheit erhöhen.

Die Behandlungsfortschritte haben eine Chronifizierung der Krankheit gefördert, die wieder zu ganz neuen Problemen führt, etwa bei der Rehabilitation, am Arbeitsplatz oder bei finanziellen Problemen. Menschen, die Beratung und Begleitung brauchen, etwa die schlechtversicherte Frau um fünfzig, die medizinisch als arbeitsfähig gilt, aber vom RAV nicht vermittelt werden kann, da die vielen Absenzen und zurückbleibende Nebenwirkungen infolge Therapien die Chancen im Stellenmarkt massiv vermindert haben.

Liselotte Dietrich findet, dass die jungen Ärzte in den letzten zehn Jahren besser ausgebildet und trotz fehlender Lebenserfahrung sensibler und kommunikativ kompetenter geworden seien. Obwohl es für junge Menschen schwierig bleibe, Emotionen und Nichtstun auszuhalten. Das berufliche Umfeld sei hektischer, die Behandlungen komplexer und die gestiegenen Ansprüche und Erwartungen der Patienten eine zusätzliche Herausforderung geworden. Ohne Teamarbeit geht nichts mehr. Gerne arbeitet sie mit Seelsorgern zusammen, aber auch mit Hausärzten, die sich vom Erfahrungsaustausch besonders angesprochen fühlten. Weiterbildung, Intervision und Supervision seien darum unverzichtbare Hilfen im anspruchsvollen Umgang mit kranken Menschen.

Weitere Auskünfte zu Frau Dietrich finden sich auf ihrer Homepage www.supervision-dietrich.ch

Dazu gehören Angebot und Beratung, aber auch Hinweise auf Publikationen zur Kunst, im Beruf gesund zu bleiben, zu Angehörigen als wichtigste Bezugspersonen von Kranken, zur gemeinsamen Entscheidungsfindung in schwierigen Situationen oder zur Erkennung von Krisen und ihrer Bewältigung. 